\title{
Subjects With Intellectual Disability and Familial Need for Full-Time Special Education Show Regional Brain Alterations: A Voxel-Based Morphometry Study
}

\author{
MINNA K. MANNERKOSKI, HANNU J. HEISKALA, KOEN VAN LEEMPUT, LAURA E. ÅBERG, RAILI RAININKO,
} JANNE HÄMÄlÄINEN, AND TAINA H. AUTTI

\begin{abstract}
Department of Gynecology and Pediatrics [M.K.M., H.J.H., L.E.Å.] and Helsinki Medical Imaging Center [K.L., J.H., T.H.A.], Helsinki
University Central Hospital, 00290 Helsinki, Finland; Department of Radiology [R.R.], Uppsala University, 75185 Uppsala, Sweden
\end{abstract}

\begin{abstract}
Subjects attending full-time special education (SE) often have multifactorial background for their cognitive impairment, and brain MRI may show nonspecific changes. As voxel-based morphometry reveals regional volume differences, we applied this method to 119 subjects with cognitive impairments and familial need for full-time SE- graded into three levels from specific disorders of cognitive processes (level 1) to intellectual disability (IQ $<70$; level 3) - and to 43 age-matched controls attending mainstream education (level 0). Subjects in SE groups had smaller global brain white matter (WM), cerebrospinal fluid, and total brain volume than controls. Compared with controls, subjects with intellectual disabilities in SE level 3 showed greater regional gray matter volumes bilaterally in the ventral and dorsal anterior cingulate cortex and smaller regional gray matter volumes in the left thalamus and cerebellar hemisphere. Further, they had greater WM volume in the left frontoparietal region and smaller WM volumes in the posterior limbs of the internal capsules. Subjects in SE level 1 and 2 groups showed the same tendency, but the results were nonsignificant. In conclusion, compared with controls, subjects with intellectual disabilities showed in voxel-based morphometry analysis several regional brain alterations.
\end{abstract}

(Pediatr Res 66: 306-311, 2009)

$I^{n}$ developed countries, approximately one of every five school-aged children needs special education (SE) of some type, and the need for full-time SE is $\sim 8 \%(1,2)$. Epidemiologic studies indicate that the etiology of intellectual disability (ID) (ICD-10 codes F70-F79) is unexplained or multifactorial in approximately half of the cases (3), a number even higher among children with specific disorders of cognitive processes (DCP). In MRI examinations, ventricular enlargements, subtle callosal abnormalities, cerebral cortical anomalies, white matter (WM) signal intensity abnormalities, and mild cerebellar fissure enlargements typically appear in subjects with ID (4-7). These findings have been designated as neuroimaging markers frequently associated with ID (5). Nevertheless, children and adolescents with IDs of unexplained etiology often

Received January 27, 2009; accepted May 19, 2009.

Correspondence: Minna K. Mannerkoski, MD, Hospital for Children and Adolescents, Helsinki University Central Hospital, P.O. Box 800, 00290 Helsinki, Finland; e-mail: minna.mannerkoski@hus.fi

Supported by the Arvo and Lea Ylppö Foundation, Helsinki, Finland; the Rinnekoti Research Foundation, Espoo, Finland; the EVO Fund of the Helsinki Medical Imaging Center, Helsinki University Central Hospital; the Children' Castle Foundation, Hospital for Children and Adolescents, Helsinki University Central Hospital; the Yrjö Jahnsson Foundation, Helsinki, Finland; and the Märta Donner Foundation, Oulu, Finland. have brain MRIs considered normal or that reveal only minor abnormalities (5).

Voxel-based morphometry (VBM) is a fully automatic method for assessing changes throughout the brain by analyzing local volume differences (8). In children and adolescents with cognitive deficits, many reports applying VBM exist (9-14). However, to our knowledge, only one VBM study to date has investigated children and adolescents with IDs of unexplained etiology (15). Compared with controls, these subjects had smaller global WM and total brain volumes and also showed several local gray matter (GM) and WM volume differences in both the cerebrum and the cerebellum (15). In intellectually intact children and adolescents, volumetric MRI studies with VBM have demonstrated a positive correlation between global GM volume and general intelligence and positive correlations between intelligence and local GM volume in many brain regions, including the frontal, temporal, and parietal lobes and the cerebellum; the correlation varies depending on the mean age of the study population $(16,17)$.

In Finland, SE groups are graded according to intellectual disability (IQ) ranges and specific DCP, and psychological evaluation precedes transfer into full-time SE groups. Consequently, SE groups included here separate subjects according to their general cognitive abilities. Our hypothesis was that subjects in full-time SE with ID or DCP of unexplained, familial etiology share similar local brain volume changes in several brain areas related to cognitive functions. We thus carried out a fully automatic analysis of MR images of subjects with ID or DCP and familial need for full-time SE and correlated alterations between SE levels and controls.

\section{METHODS}

Patients. The study design and patient recruitment have been previously published (18). Briefly, from the 4500 pupils comprising full-time SE groups in the Uusimaa District, Finland, a sample of 900 children was collected in random order. Of these, 119 siblings (mean age $11.9 \mathrm{y}$, SD 3.9; range 5.6 to 29.5; 73 boys) met the inclusion criteria (attending or had attended one of the predefined full-time SE groups and had at least one sibling also in a full-time SE group, etiology unexplained in both, i.e., the cause of the ID or DCP for each subject remained unspecified in medical investigations performed to

\footnotetext{
Abbreviations: ACC, anterior cingulate cortex; CSF, cerebrospinal fluid, DCP, disorders of cognitive processes; ID, intellectual disability; SE, special education; VBM, voxel-based morphometry
} 
date) and underwent MRI. From each family, all siblings with need for full-time SE were included in the study (number of siblings ranged from 2 to 4). The SE groups were categorized into three levels: in level $1(n=49$; mean age 10.9 y, SD 3.9; range 5.9 to 17.3), subjects had specific DCP, such as problems in speech and language development, attention, sensory motor skills, or mathematics, and they followed mainly the mainstream education syllabus in small-sized classes; in level $2(n=40$; mean age 12.2, SD 2.9; range 5.3 to 17.7 ), subjects with borderline to mild ID had all courses in the adjusted education syllabus; and in level $3(n=30$; mean age 12.1, SD 5.7; range 5.4 to 29.5 ), subjects with mild to severe ID were trained in daily activities according to each subject's abilities. Although the IQ distribution between various levels was not mutually exclusive, in level 3, the IQ of all subjects was below 70. The control group was categorized as level 0 and consisted of 43 pupils (mean age 12.0 y, SD 3.1; range 6.1 to 18.6; 26 boys) participating in mainstream education. The parents gave informed consent, and Ethics Committee of the Rinnekoti Foundation and Ministry of Social Affairs and Health in Finland approved the study protocol. Clinical data and results of visual MRI analysis of study subjects in SE have been previously published (18). In SE level 3, MRI findings existed more often than in other groups and included thin corpus callosum and enlarged supra- and infratentorial cerebrospinal fluid (CSF) spaces. Controls were recruited for imaging purposes only.

MRI examinations. All subjects underwent brain MRI at 1.5T (Siemens Magnetom Vision; Siemens, Erlangen, Germany). We obtained T2-weighted axial slices with a fast spin-echo sequence (TR/TE 3000/85, $14 \mathrm{ms,}$, with slice thickness $5 \mathrm{~mm}$, gap $1.5 \mathrm{~mm}$ ), an axial fluid attenuated inversion recovery (FLAIR) sequence (TR/TE/T1 9999/105/2500 ms, with slice thickness $5 \mathrm{~mm}$, gap $1.5 \mathrm{~mm}$ ), and a sagittal three-dimensional magnetization-prepared rapid acquisition gradient echo (MPRAGE) sequence (TR/TE/T1 9.7/4.0/20 ms, with slice thickness $1 \mathrm{~mm}$, no gap). For all sequences, matrix was $256 \times 256$ and field-of-view 230.

After independent visual evaluation by two experienced radiologists (T.A. and R.R.), we excluded the images of 10 subjects from VBM analyses (three had extensive motion artifacts, two had extensive cerebral volume loss, and five lacked some of the required 170 MPRAGE slices). Thus, 152 subjects (39 in level 0, 49 in level 1, 38 in level 2, and 26 in level 3) underwent the final VBM analyses.

Image processing and statistical analysis. We analyzed our data with the SPM2 software package (http://www.fil.ion.ucl.ac.uk/spm), using a VBM approach (8) to identify brain regions significantly related to SE level. VBM assesses changes throughout the brain, without requiring prior hypotheses of potential areas of interest, by analyzing local differences in segmentations automatically obtained. The standard VBM protocol is described in detail by Ashburner and Friston (8); here, we summarize the main steps.

First, an anatomical template is created that is representative of all MR images used in the study. By a nonrigid registration technique (see details below), each image is spatially normalized to the ICBM 152 template [Montreal Neurologic Institute (MNI), Quebec, Canada], which is derived from 152 subjects and approximates the Talairach space. The images are then resampled onto a common image grid, smoothed with a Gaussian kernel, and averaged to create a mean (template) image. Second, all images are transformed to the same stereotactic space by nonlinearly coregistering them with the previously created template and resampling them onto a common image grid. The nonrigid registration consists of an initial affine registration, followed by a nonlinear deformation constructed as a linear combination of smooth basis functions. Third, each image is segmented into WM, GM, CSF, and nonbrain tissue by a Gaussian mixture classifier that explicitly models intensity inhomogeneities caused by the MR acquisition process. The segmentation process relies on prior probabilities for distribution of tissue types throughout the brain, derived from a large number of segmentations of normal subjects. Finally, the segmentation images are smoothed by convoluting with a Gaussian kernel and subjected to statistical analysis (see below). The smoothing step helps to overcome local shape differences not removed by the nonlinear registration and additionally renders the data more conformable to the assumptions made in subsequent statistical analyses $(8,19)$.
For our analysis, we adopted the optimized VBM scheme proposed by Good et al. (19), which is an improvement upon the standard VBM protocol in a number of ways. We created study-specific prior probabilities for WM, GM, and CSF by averaging the segmentations of all study subjects obtained with the standard VBM protocol. We then used the resulting priors, which better reflected the characteristics of our study population, to obtain improved segmentations. We also corrected segmentation errors by removing unconnected nonbrain voxels and calculated all nonrigid registrations by first segmenting each image after affine normalization only and subsequently calculating the nonlinear deformation by coregistering the resulting GM segmentation with the GM prior probability map. Thus, nonbrain voxels do not contribute to the estimation of the deformation field, affording optimal spatial normalization in the brain tissues. Furthermore, we modulated the segmentation images by the Jacobian determinants of the deformation fields used to drive each image into the stereotactic space. This step ensures that the original amount of tissue is preserved within each structure. As a result, any differences found with VBM can be interpreted as local differences in absolute tissue volume.

We assessed regional relationships between WM and GM volume between controls in ME and subjects in full-time SE by group-wise comparisons (controls versus level 1; controls versus level 2; and controls versus level 3) by a general linear model that included SE level as a factor and gender, age, and global mean voxel value (WM globals for the WM analysis and GM globals for the GM analysis) as confounding covariates. The reason for including global mean voxel values as confounding covariates is that this allows us to assess regionally specific changes within the white/gray matter compartments "over and above" global white/gray matter changes, which we report separately. To correct for multiple comparisons, we used Gaussian random field theory. Significance levels for one-sided $t$-statistics were set at $p<0.025$ (positive and negative effects were studied).

\section{RESULTS}

Global brain volume differences. For subjects attending $\mathrm{SE}$, the higher their SE group level, the smaller their total brain volume ( $p=0.013$, Table 1$)$. They also showed similar negative correlations between global WM $(p=0.002)$ and CSF $(p=0.033)$ volumes and their SE group level; however, compared with controls, no significant differences in global GM volumes existed $(p=0.110)$.

Regional brain volume differences. Table 2 presents the MNI coordinates and cluster sizes for the regional GM and WM volume differences detected between level 3 subjects and controls. Subjects with IDs attending SE level 3 showed greater regional GM volumes than controls bilaterally in the ventral and dorsal anterior cingulate cortex (ACC) (Fig. 1). Compared with controls, subjects in SE level 3 showed smaller local GM volume in the left cerebellar hemisphere and the left thalamus (Fig. 2); greater local WM volume in the left frontoparietal region (Fig. 3); and smaller local WM volumes (Fig. 4) bilaterally in the posterior limbs of the internal capsules. Noteworthy is that compared with controls, subjects in SE level 1 and 2 groups showed similar gray and WM volume differences in corresponding regional brain areas; however, the changes were smaller than for subjects in level 3 and thus remained nonsignificant. The study groups showed no differences in regional CSF volumes.

Table 1. Mean global GM, WM, CSF, and total brain volumes of controls (level 0) and subjects with disorders of cognitive processes or intellectual disabilities and familial need for full-time special education (levels 1-3)

\begin{tabular}{|c|c|c|c|c|c|}
\hline & Level $0(n=43)$ & Level $1(n=49)$ & Level $2(n=41)$ & Level $3(n=32)$ & $p$ \\
\hline Mean GM volume (SD), mL & 779 (73.8) & $760(69.5)$ & $741(90.4)$ & $730(130.5)$ & 0.110 \\
\hline Mean WM volume (SD), mL & $431(36.6)$ & $403(51)$ & $403(59.9)$ & $379(66)$ & 0.002 \\
\hline Mean CSF volume (SD), mL & $620(89)$ & $584(90.4)$ & $575(110.5)$ & $550(107.2)$ & 0.033 \\
\hline Mean total volume (SD), $\mathrm{mL}$ & $1829(174)$ & 1747 (177) & $1719(238)$ & $1656(268)$ & 0.013 \\
\hline
\end{tabular}

Statistical analysis calculated by univariate analysis of variance, controlling for age and sex. 
Table 2. VBM local volume differences between the subjects with intellectual disability and controls

\begin{tabular}{|c|c|c|c|c|c|c|}
\hline \multirow[b]{2}{*}{ Region } & \multicolumn{3}{|c|}{ MNI coordinates } & \multirow[b]{2}{*}{ Cluster size } & \multirow[b]{2}{*}{$T_{\text {value }}$} & \multirow[b]{2}{*}{ Adjusted $p$ value } \\
\hline & $x$ & $Y$ & $z$ & & & \\
\hline Greater GM volume & & & & & 4.51 & $<0.001$ \\
\hline Ventral ACC & 12 & 36 & -2 & 2496 & & \\
\hline Dorsal ACC left & -11 & -39 & 32 & 1107 & & \\
\hline Dorsal ACC right & 11 & -47 & 28 & 666 & & \\
\hline Smaller GM volume & & & & & 4.51 & $<0.001$ \\
\hline Left cerebellum & -11 & -54 & -15 & 4062 & & \\
\hline Left thalamus & -8 & -14 & 15 & 210 & & \\
\hline Greater WM volume & & & & & 4.32 & $<0.001$ \\
\hline Left frontaparietal region & -18 & -24 & 49 & 3099 & & \\
\hline Smaller WM volume & & & & & 4.33 & $<0.001$ \\
\hline Posterior internal capsule left & -21 & -31 & 10 & 141 & & \\
\hline Posterior internal capsule right & 20 & -26 & 9 & 815 & & \\
\hline
\end{tabular}

Maximum coordinates in Montreal Neurological Institute (MNI) stereotactic space.

A

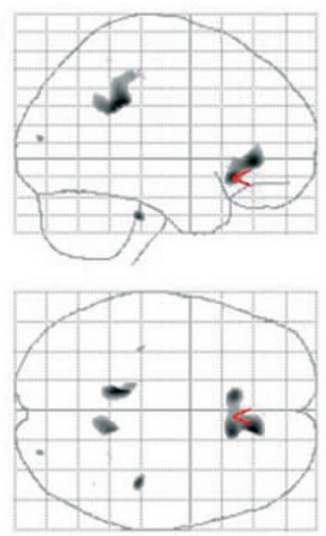

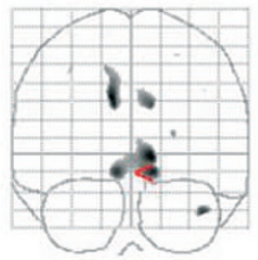
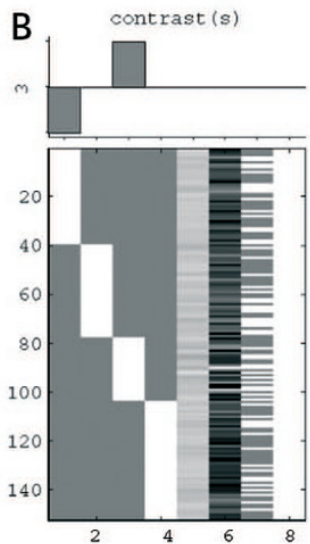

C
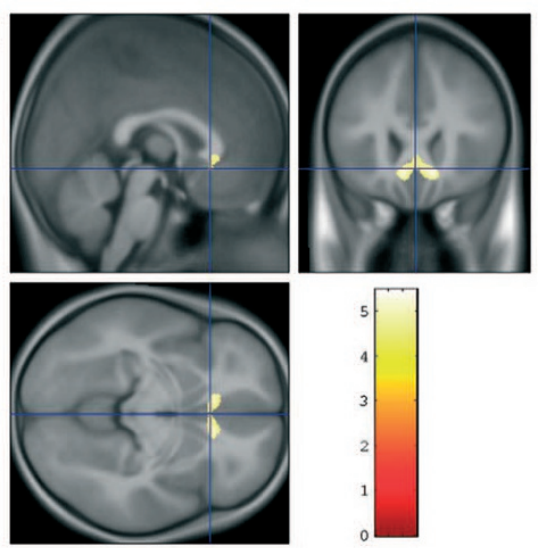

Figure 1. Gray matter enlargements in anterior cingulate cortex of subjects with ID attending SE level 3, compared with controls. Voxels with significant positive correlation in local tissue volume ( $p<0.025$, corrected for multiple comparisons) are shown. $A$, all the positively correlated voxels projected through three main directions; $B$, the design matrix; $C$, voxels in the anterior cingulate cortex overlaid on the average of the spatially normalized MR scans of all subjects.

A
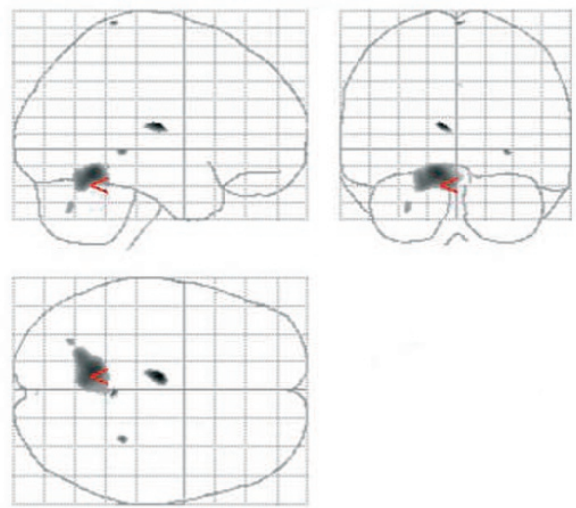
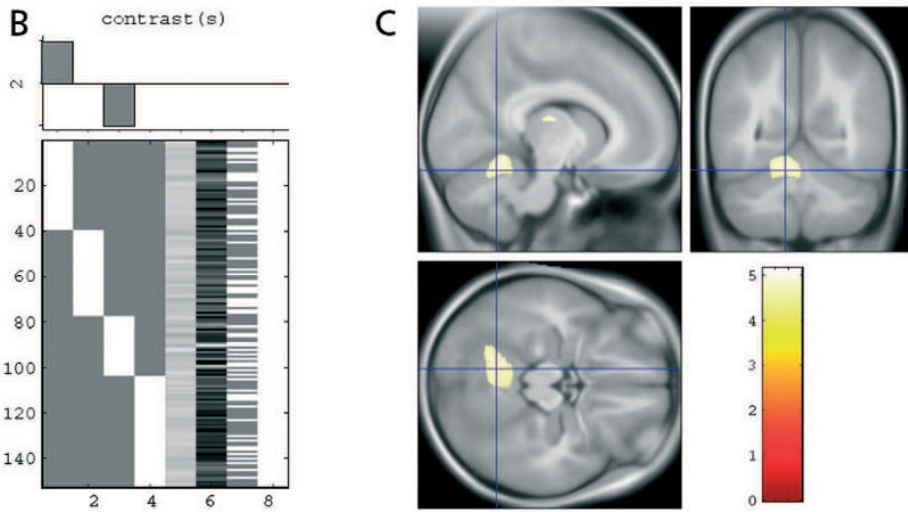

Figure 2. Gray matter shrinkage in left cerebellar hemisphere and left thalamus of subjects with ID attending SE level 3, compared with controls. Voxels with significant negative correlation in local tissue volume ( $p<0.025$, corrected for multiple comparisons) are shown. $A$, all the positively correlated voxels projected through three main directions; $B$, the design matrix; $C$, voxels in left cerebellar hemisphere overlaid on the average of spatially normalized MR scans of all subjects.

\section{DISCUSSION}

In this volumetric study, we have demonstrated that in subjects with ID or DCP and a familial need for full-time $\mathrm{SE}$, the mean global $\mathrm{WM}$ and total brain volumes were smaller than in controls attending mainstream education. This correlation was negative; in other words, those in the highest (i.e., training education) SE level with ID had the smallest global volumes. Correspondingly, in recent studies of intellectually intact subjects, both the global WM volume and the total brain volume correlated positively with IQ $(16,17,20)$. Moreover, these studies also demonstrated a positive correlation between global GM volume and IQ. In 
A
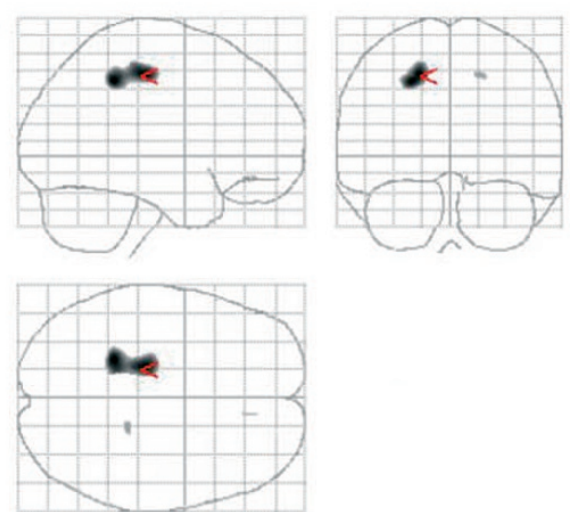

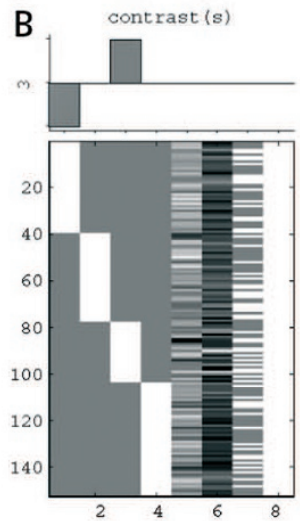

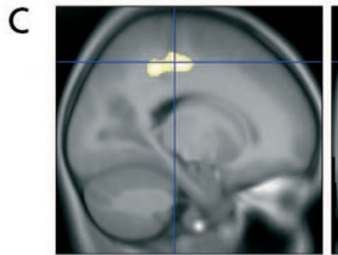
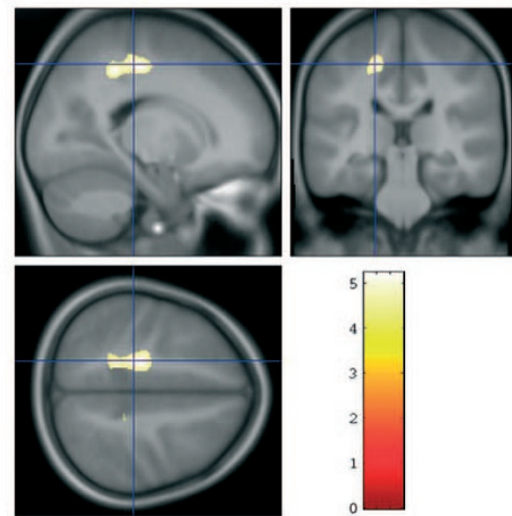

Figure 3. White matter enlargements in left frontoparietal region of subjects with ID attending SE level 3, compared with controls. Voxels with significant positive correlation in local tissue volume ( $p<0.025$, corrected for multiple comparisons) are shown. A, all the positively correlated voxels projected through three main directions; $B$, the design matrix; $C$, voxels in left frontoparietal region overlaid on the average of spatially normalized MR scans of all subjects.

A
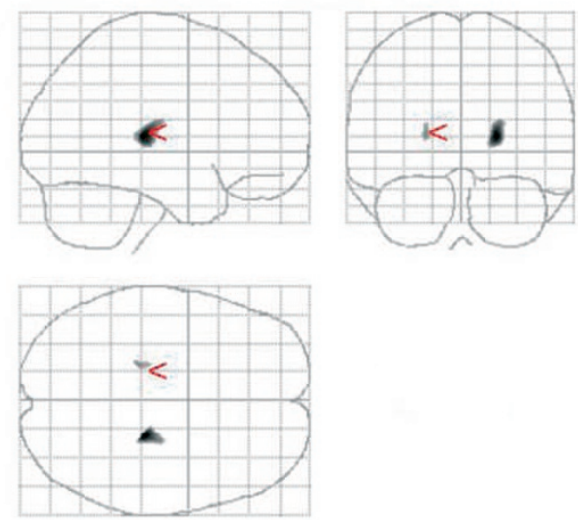
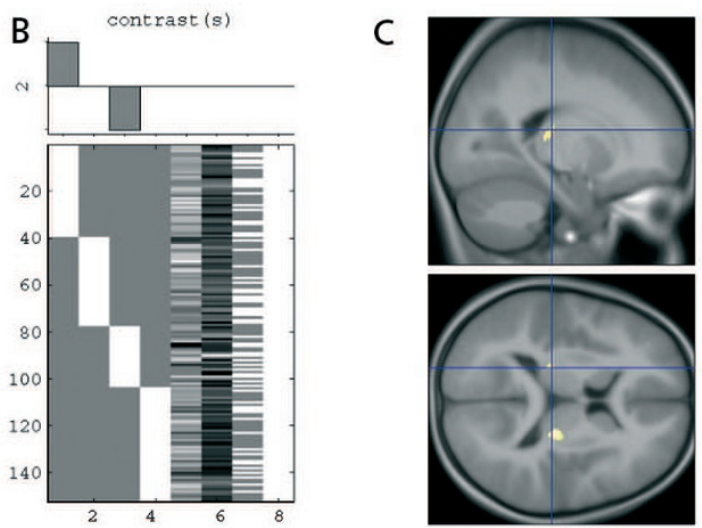
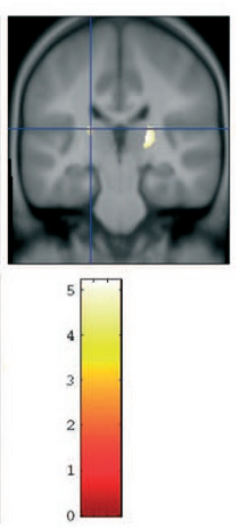

Figure 4. White matter shrinkage bilaterally in posterior limbs of the internal capsules of subjects with ID attending SE level 3, compared with controls. Voxels with significant negative correlation in local tissue volume $(p<0.025$, corrected for multiple comparisons) are shown. $A$, all the positively correlated voxels projected through three main directions; $B$, the design matrix; $C$, voxels the in posterior limbs of the internal capsules overlaid on the average of spatially normalized MR scans of all subjects.

fact, in our study, the mean global GM volumes were smaller in subjects in SE than in controls, although the differences remained nonsignificant. Similarly to our results, in a previous study of intellectually disabled adolescents, both global WM and total brain volumes, but not global GM volume, were smaller in subjects than in controls (15).

In addition to global volume differences, we demonstrated several regional volume differences between subjects with ID (level 3) and controls. Compared with controls, findings for level 3 subjects included greater GM volume in the anterior cingulate cortex; smaller GM volume in the left superior, medial cerebellar hemisphere, and left thalamus; greater WM volume in the left frontoparietal region; and smaller WM volume in both posterior internal capsules. The left-sided alterations were on the dominant cerebral hemisphere because a great majority of these subjects were right-handed. However, we were unable to include handedness in covariance analysis because the handedness was not clear in all of the subjects in SE. Noteworthy is that in a large volumetric study of the effects of sex and handedness on brain structure, no regional main effects of handedness were revealed (21).
According to data on previous cross-sectional, volumetric reports of brain development in childhood and adolescence, general intelligence seems to correlate positively with the volume of various cortical regions depending on the mean age of the study population. For younger children, the peak correlation exists in the anterior cingulate cortex (16), whereas for adolescents, these regions include the orbitofrontal and the medial prefrontal cortex and the cerebellum (17), and for adults, additionally the dorsal prefrontal cortex (22). Moreover, longitudinal reports show the development of the cortex as a complex and dynamic process, with differing maturational patterns for subjects with superior, high, and average levels of general intelligence (23-25). In fact, according to Shaw et al. (25), the correlation between intelligence and cortical thickness (mainly in prefrontal area) changes from negative in early childhood to positive in later childhood and adolescence. A delay in regional cortical maturation of subjects with ADHD was recently published (26). Although this study was not longitudinal, the greater GM volume in the cingulate cortex of subjects with ID and a familial need for full-time SE may be due to disturbance in the normal maturation. Abnormalities in 
maturational processes are most likely linked to an underlying genetic background because we investigated siblings with ID or DCP of unexplained etiologies. Twin studies suggest that global GM and WM volumes and regional cortical GM volumes are highly genetically determined $(20,27)$.

The ACC is under intense neuroimaging investigation and has thus far proven to be a very versatile area. Nelson et al. (28) showed the ACC to activate specifically when subjects were performing conflicting stimulus-response workingmemory tasks. Furthermore, the ACC is involved in maintaining attention and motivation and in many processes such as working and spatial memory, adaptive behavior, cognitive fluency, making optimal decisions, and monitoring response conflict, errors, and unfavorable outcomes, all essentially associated with learning (29-32). As the ACC is activated in numerous situations of cognitive processes and learning, disturbed activity-dependent plasticity may also be involved $(33,34)$.

Some examples of assumed activity-dependent plasticity changes detected in MRI studies exist. In Tourette syndrome, a volumetric study showed greater GM volume bilaterally in the ventral putamen than in controls (35), and a PET study suggested overactive basal ganglia in subjects with Tourette (36). In dyslexic boys, an fMRI study showed overactivation of the precentral gyrus (37), and a voxel-by-voxel analysis of adult dyslexics revealed increased GM density in the corresponding area (38). In intellectually intact subjects, an example of activity-dependent plasticity changes leading to altered regional brain volumes includes local training-induced GM increases in persons learning to juggle (39).

A diffusion tensor MRI study of human brain maturation showed that the thalami and posterior internal capsules reach the $90 \%$ maturational stage only in the early twenties (40). Maturational abnormalities could therefore explain the smaller GM and WM volumes of the left thalami and the posterior internal capsules in our subjects in SE level 3. Thalamic alterations have been reported in subjects with cognitive impairments (14), and in healthy adolescents, general IQ correlated positively with GM volume in the thalamus (17).

Visual analysis of the brain MRI of our subjects in SE level 3 revealed that they possessed, unlike controls, enlarged cerebellar fissures (18). Therefore, the smaller GM volume of the cerebellum detected by VBM was expected. Previously, a weak correlation between total cerebellar volume and IQ has been described (41), and the role of the cerebellum in various cognitive processes acknowledged $(42,43)$.

In our study, the greater WM volumes in subjects in SE were located in the posterior frontal and anterior parietal regions. The smaller total brain WM volume has been suggested to indicate delayed myelination in children with global developmental delays (44), and while diffuse WM abnormalities for children with a single domain developmental delay have been described (45), reports of regional WM alterations related to cognitive impairment and learning in children are rare. One of these reports a frontoparietal WM enlargement in children with developmental language disorder (46).

Our population-based setting, a sufficiently large population, and a low number of subjects with severe visible abnor- malities resulting in exclusion (18) enabled reliable VBM analyses. In SE level 3, the overall CSF volumes were smaller than in controls, although in visual analysis they more often than controls had enlarged CSF spaces. However, subjects with the most extensively enlarged CSF spaces were excluded from the VBM analysis.

Limitations. This study has, however, some drawbacks that limit generalization of the results. There is a lack of information on individual general IQs, which could have served as a dependent variable in the analyses. Instead, we chose SE level, which, in addition to placing the child in a certain IQ range, tells us the functional level of the child in the school environment. At level 3, because two siblings (a twin pair) were born preterm at gestation week 29 , their prematurity may explain at least part of their mental impairment. Their visual MRI evaluations were, however, normal and showed no signs of the ischemic changes often associated with prematurity. Because these twins had an older brother with a similar mental impairment who was born at term, they were considered to have an unknown shared cause. Siblings in SE had a greater age range than controls due to inclusion of two adult siblings (one 24-y-old and one 29-y-old) in SE level 3.

We have reported statistical group differences in both global white and gray matter volumes, as well as regional differences above and beyond such global changes. Although our procedure for normalizing out global volumes from regional volume changes (by including them as confounding covariates in each local general linear model) is standard practice in VBM analysis (19), global normalization is known to be a thorny issue in neuroimage analysis $(47,48)$. In particular, because we have calculated global effects by integrating regional volume measurements across the entire brain, global and regional effects may be confounded, thereby reducing the sensitivity of our method for detecting true local volume changes (47). More robust global measures may be obtained by examining only brain regions that are relatively unaffected (47), but because knowing what these regions are in turn requires the outcome of a morphometric study, we have not pursued this option further.

In conclusion, compared with controls in mainstream education, subjects with ID from families with two or more siblings in full-time SE showed several regional volume differences in brain areas known to be involved in various cognitive processes. Further investigations with fMRI and diffusion tensor imaging fiber tractography are warranted to explore both the function and the structure of these areas in children and adolescents in full-time SE.

Acknowledgments. We thank all the families, especially all of the siblings in SE, and all controls who participated in this study.

\section{REFERENCES}

1. Central Statistical Office of Finland 2008 Available at: www.tilastokeskus.fi/tk/he/ oppilaitostilastot_erityisopetus. Accessed June 10, 2008

2. Avchen RN, Scott KG, Mason CA 2001 Birth weight and school-age disabilities: a population-based study. Am J Epidemiol 154:895-901

3. Cans C, Wilhelm L, Baille MF, du Mazaubrun C, Grandjean H, Rumeau-Rouquette C 1999 Aetiological findings and associated factors in children with severe mental retardation. Dev Med Child Neurol 41:233-239 
4. Soto-Ares G, Joyes B, Lemaitre MP, Vallee L, Pruvo JP 2003 MRI in children with mental retardation. Pediatr Radiol 33:334-345

5. Decobert F, Grabar S, Merzoug V, Kalifa G, Ponsot G, Adamsbaum C, des Portes V 2005 Unexplained mental retardation: is brain MRI useful? Pediatr Radiol 35:587-596

6. Spencer MD, Gibson RJ, Moorhead TW, Keston PM, Hoare P, Best JJ, Lawrie SM, Johnstone EC 2005 Qualitative assessment of brain anomalies in adolescents with mental retardation. AJNR Am J Neuroradiol 26:2691-2697

7. Widjaja E, Nilsson D, Blaser S, Raybaud C 2008 White matter abnormalities in children with idiopathic developmental delay. Acta Radiol 49:589-595

8. Ashburner J, Friston KJ 2000 Voxel-based morphometry-the methods. Neuroimage 11:805-821

9. Overmeyer S, Bullmore ET, Suckling J, Simmons A, Williams SC, Santosh PJ, Taylor E 2001 Distributed grey and white matter deficits in hyperkinetic disorder: MRI evidence for anatomical abnormality in an attentional network. Psychol Med $31: 1425-1435$

10. Sowell ER, Thompson PM, Mattson SN, Tessner KD, Jernigan TL, Riley EP, Toga AW 2001 Voxel-based morphometric analyses of the brain in children and adolescents prenatally exposed to alcohol. Neuroreport 12:515-523

11. Isaacs EB, Edmonds CJ, Lucas A, Gadian DG 2001 Calculation difficulties in children of very low birthweight: a neural correlate. Brain 124:1701-1707

12. Boddaert N, Chabane N, Gervais H, Good CD, Bourgeois M, Plumet MH, Barthelemy C, Mouren MC, Artiges E, Samson Y, Brunelle F, Frackowiak RS, Zilbovicius M 2004 Superior temporal sulcus anatomical abnormalities in childhood autism: a voxel-based morphometry MRI study. Neuroimage 23:364-369

13. Carmona S, Vilarroya O, Bielsa A, Tremols V, Soliva JC, Rovira M, Tomas J, Raheb C, Gispert JD, Batlle S, Bulbena A 2005 Global and regional gray matter reductions in ADHD: a voxel-based morphometric study. Neurosci Lett 389:88-93

14. Boddaert N, Mochel F, Meresse I, Seidenwurm D, Cachia A, Brunelle F, Lyonnet S, Zilbovicius M 2006 Parieto-occipital grey matter abnormalities in children with Williams syndrome. Neuroimage 30:721-725

15. Spencer MD, Moorhead TW, Lymer GK, Job DE, Muir WJ, Hoare P, Owens DG, Lawrie SM, Johnstone EC 2006 Structural correlates of intellectual impairment and autistic features in adolescents. Neuroimage 33:1136-1144

16. Wilke M, Sohn JH, Byars AW, Holland SK 2003 Bright spots: correlations of gray matter volume with IQ in a normal pediatric population. Neuroimage 20:202-215

17. Frangou S, Chitins X, Williams SC 2004 Mapping IQ and gray matter density in healthy young people. Neuroimage 23:800-805

18. Mannerkoski M, Heiskala H, Raininko R, Åberg L, Sarna S, Wirtavuori K, Autti T 2009 Brain MRI of siblings from families with two or more children with learning and intellectual disabilities and need for full-time special education. Acta Radiol 50:437-445

19. Good CD, Johnsrude IS, Ashburner J, Henson RN, Friston KJ, Frackowiak RS 2001 A voxel-based morphometric study of ageing in 465 normal adult human brains. Neuroimage 14:21-36

20. Posthuma D, De Geus EJ, Baare WF, Hulshoff Pol HE, Kahn RS, Boomsma DI 2002 The association between brain volume and intelligence is of genetic origin. Nat Neurosci 5:83-84

21. Good CD, Johnsrude I, Ashburner J, Henson RN, Friston KJ, Frackowiak RS 2001 Cerebral asymmetry and the effects of sex and handedness on brain structure: voxel-based morphometric analysis of 465 normal adult human brains. Neuroimage 14:685-700

22. Haier RJ, Jung RE, Yeo RA, Head K, Alkire MT 2004 Structural brain variation and general intelligence. Neuroimage 23:425-433

23. Giedd JN, Blumenthal J, Jeffries NO, Castellanos FX, Liu H, Zijdenbos A, Paus T, Evans AC, Rapoport JL 1999 Brain development during childhood and adolescence: a longitudinal MRI study. Nat Neurosci 2:861-863

24. Gogtay N, Giedd JN, Lusk L, Hayashi KM, Greenstein D, Vaituzis AC, Nugent TF 3rd, Herman DH, Clasen LS, Toga AW, Rapoport JL, Thompson PM 2004 Dynamic mapping of human cortical development during childhood through early adulthood. Proc Natl Acad Sci USA 101:8174-8179
25. Shaw P, Greenstein D, Lerch J, Clasen L, Lenroot R, Gogtay N, Evans A, Rapoport J, Giedd J 2006 Intellectual ability and cortical development in children and adolescents. Nature 440:676-679

26. Shaw P, Eckstrand K, Sharp W, Blumenthal J, Lerch JP, Greenstein D, Clasen L, Evans A, Giedd J, Rapoport JL 2007 Attention-deficit/hyperactivity disorder is characterized by a delay in cortical maturation. Proc Natl Acad Sci USA 104:19649_ 19654

27. Thompson PM, Cannon TD, Narr KL, van Erp T, Poutanen VP, Huttunen M, Lonnqvis J, Standertskjold-Nordenstam CG, Kaprio J, Khaledy M, Dail R, Zoumalan CI, Toga AW 2001 Genetic influences on brain structure. Nat Neurosci 4:1253-1258

28. Nelson JK, Reuter-Lorenz PA, Sylvester CY, Jonides J, Smith EE 2003 Dissociable neural mechanisms underlying response-based and familiarity-based conflict in working memory. Proc Natl Acad Sci USA 100:11171-11175

29. Paus T 2001 Primate anterior cingulate cortex: where motor control, drive and cognition interface. Nat Rev Neurosci 2:417-424

30. Pujol J, Lopez A, Deus J, Cardoner N, Vallejo J, Capdevila A, Paus T 2002 Anatomical variability of the anterior cingulate gyrus and basic dimensions of human personality. Neuroimage 15:847-855

31. Bush G, Vogt BA, Holmes J, Dale AM, Greve D, Jenike MA, Rosen BR 2002 Dorsal anterior cingulate cortex: a role in reward-based decision making. Proc Natl Acad Sci USA 99:523-528

32. Kennerley SW, Walton ME, Behrens TE, Buckley MJ, Rushworth MF 2006 Optimal decision making and the anterior cingulate cortex. Nat Neurosci 9:940-947

33. Penn AA, Shatz CJ 1999 Brain waves and brain wiring: the role of endogenous and sensory-driven neural activity in development. Pediatr Res 45:447-458

34. Johnston MV 2004 Clinical disorders of brain plasticity. Brain Dev 26:73-80

35. Ludolph AG, Juengling FD, Libal G, Ludolph AC, Fegert JM, Kassubek J 2006 Grey-matter abnormalities in boys with Tourette syndrome: magnetic resonance imaging study using optimised voxel-based morphometry. Br J Psychiatry 188:484-485

36. Stern E, Silbersweig DA, Chee KY, Holmes A, Robertson MM, Trimble M, Frith CD, Frackowiak RS, Dolan RJ 2000 A functional neuroanatomy of tics in Tourette syndrome. Arch Gen Psychiatry 57:741-748

37. Corina DP, Richards TL, Serafini S, Richards AL, Steury K, Abbott RD, Echelard DR, Maravilla KR, Berninger VW 2001 fMRI auditory language differences between dyslexic and able reading children. Neuroreport 12:1195-1201

38. Vinckenbosch E, Robichon F, Eliez S 2005 Gray matter alteration in dyslexia: converging evidence from volumetric and voxel-by-voxel MRI analyses. Neuropsychologia 43:324-331

39. Draganski B, Gaser C, Busch V, Schuierer G, Bogdahn U, May A 2004 Neuroplasticity: changes in grey matter induced by training. Nature 427:311-312

40. Lebel C, Walker L, Leemans A, Phillips L, Beaulieu C 2008 Microstructural maturation of the human brain from childhood to adulthood. Neuroimage 40:1044-1055

41. Paradiso S, Andreasen NC, O'Leary DS, Arndt S, Robinson RG 1997 Cerebellar size and cognition: correlations with IQ, verbal memory and motor dexterity. Neuropsychiatry Neuropsychol Behav Neurol 10:1-8

42. Wassmer E, Davies P, Whitehouse WP, Green SH 2003 Clinical spectrum associated with cerebellar hypoplasia. Pediatr Neurol 28:347-351

43. Steinlin M 2007 The cerebellum in cognitive processes: supporting studies in children. Cerebellum 6:237-241

44. Pujol J, Lopez-Sala A, Sebastian-Galles N, Deus J, Cardoner N, Soriano-Mas C, Moreno A, Sans A 2004 Delayed myelination in children with developmental delay detected by volumetric MRI. Neuroimage 22:897-903

45. Filippi CG, Lin DD, Tsiouris AJ, Watts R, Packard AM, Heier LA, Ulug AM 2003 Diffusion-tensor MR imaging in children with developmental delay: preliminary findings. Radiology 229:44-50

46. Herbert MR, Ziegler DA, Makris N, Filipek PA, Kemper TL, Normandin JJ, Sander HA, Kennedy DN, Caviness VS Jr 2004 Localization of white matter volume increase in autism and developmental language disorder. Ann Neurol 55:530-540

47. Petersson KM, Nichols TE, Poline JB, Holmes AP 1999 Statistical limitations in functional neuroimaging. I. Non-inferential methods and statistical models. Philos Trans R Soc Lond B Biol Sci 354:1239-1260

48. Gründer G 2009 "Absolute" or "relative": choosing the right outcome measure in neuroimaging. Neuroimage 45:258-259 